\title{
Autoorganización, autopoiesis y causalidad no lineal
}

\section{Self-organization, autopoiesis and nonlinear causality}

Nancy Carolina Castillo López

Universidad de San Carlos de Guatemala

ncastillodelinares@yahoo.com

https://orcid.org/0000-0003-0079-1466

Recibido: $26 / 06 / 2021$

Aceptado: 27/07/2021

\section{Referencia del artículo}

Castillo López , N. C. (2021). Autoorganización, autopoiesis y causalidad no lineal. Revista Diversidad Científica, 1(1). 61-69.

DOI: https://doi.org/10.36314/diversidad.v1i1.8

\section{Resumen}

El cambio paradigmático de una visión de la realidad desde la física clásica, hacia las ciencias de la vida es esencial, en virtud que la primera, a pesar de sentar las bases para explicar muchos fenómenos naturales, se queda corta cuando incluimos lo vivo en la ecuación. Reconocer los procesos autoorganizados de las diferentes estructuras, comprender los patrones de vida, y especialmente identificar sus diferencias, nos posibilitan actuaciones más asertivas, consecuentes con el ecosistema y su funcionamiento. Un punto importante de mencionar es que cuando surgió el término autopoiesis, no se aceptaba su uso para explicar los patrones de las redes de tercer orden (agregados de organismos), sin embargo, con el tiempo se ha aplicado y adaptado, reconociendo que en estos el funcionamiento depende de la relación de sus partes y no de la autopoiesis de sus componentes. Dicha situación es determinante para establecer diferencias clave como la restricción de la creatividad que imponen los organismos vivos a sus miembros contrastada con la amplificación de la misma que requieren las estructuras sociales. Lo anterior se evidencia en como las sociedades opresivas o rígidas llegan a marchitar el espíritu humano, al punto de impedir el progreso de sus integrantes.

Palabras clave: autoorganización, autopoiesis, no linealidad, estructuras disipativas 


\begin{abstract}
The paradigmatic change of a vision of reality from classical physics, to life sciences is essential, since the former, being that the first, lays the foundations to explain many natural phenomena, falls short when we include the living in the equation. Recognizing the self-organized processes of the different structures, understanding life patterns, and especially identifying their differences, allow us to perform more assertive actions, consistent with the ecosystem and its functioning. An important point to mention is that when the term autopoiesis arose, its use to explain the patterns of third-order networks (aggregates of organisms) was not accepted, however, over time it has been applied and adapted, recognizing that in these the functioning depends on the relationship of its parts and not on the autopoiesis of its components. This situation is decisive for establishing key differences such as the restriction of creativity imposed by living organisms on their members, contrasted with the amplification of creativity required by social structures. This is evidenced in how oppressive or rigid societies come to wither the human spirit, to the point of impeding the progress of its members.
\end{abstract}

Keywords: self-organization, autopoiesis, non-linearity, dissipative structures 


\section{Introducción}

El cambio paradigmático de una visión de la realidad desde la física clásica, hacia las ciencias de la vida es esencial, en virtud que la primera, a pesar de sentar las bases para explicar muchos fenómenos naturales, se queda corta cuando incluimos lo vivo en la ecuación. Estudiar el funcionamiento de los patrones, estructuras y procesos vitales, nos posibilita una mayor comprensión del mundo que, a la vez nos faculta para poder responder mejor ante los fenómenos y la incertidumbre.

El proceso para la determinación del patrón de vida, está compuesto de varias propuestas, partiendo desde la organización, refinándose más tarde en el término autoorganización, el cual sirvió de base para el establecimiento de la nueva termodinámica no lineal de llya Prigogine (1997) quien introduce el concepto de estructuras disipativas. Todo ello, fue crucial para que más adelante Maturana y Varela (1998) pudieran establecer un término más especifico como es la autopoiesis que en esencia significa auto creación.

La característica clave en este punto es la causalidad no lineal, que marca un hito en el estudio y comprensión de los fenómenos complejos, y pone de manifiesto la necesidad de una visión diferente a la convencional a fin de responder a la realidad, y dejar de lado los acercamientos teóricos que se enfocan en factores acotados y limitantes que simplifican, para dejar paso a lo complejo de la vida. Parte de la autoorganización es la adaptación a las circunstancias ambientales (Prigogine, 1997, p. 49), es decir que para poder sobrevivir como especie se deben ir generando los cambios que lo permitan. Aprender del pasado y evolucionar a nuevas formas de vida mejores, más plenas y armónicas. Comprender el funcionamiento de los organismos y las diferencias con el de las sociedades o sistemas de tercer orden también puede abrir puertas para la búsqueda de soluciones creativas a los problemas ambientales y sociales que, a pesar de los avances tecnológicos mundiales, siguen latentes siendo uno de los principales la gran desigualdad económico social que se vive.

\section{Autoorganización, estructuras disipativas y autopoiesis}

La autoorganización, surge del refinamiento que sufre el término organización, y en esencia implica espontaneidad, novedad, no linealidad y estar alejado del equilibrio. "Foerster a finales de los años cincuenta, se convirtió en el principal catalizador de la idea" como lo afirma (Capra, 1998, p. 102). Como su nombre lo indica la autoorganización comprende los procesos propios de una estructura que disipa energía, 
y que a través de bucles de retroalimentación internos mantiene su estabilidad estructural. Los procesos autoorganizados se dan en los seres vivos, así como pueden darse en fenómenos no vivos. Prigogine desarrolló una nueva termodinámica no-lineal para describir el fenómeno de la autoorganización en sistemas abiertos alejados del equilibrio según (Capra, 1998, p. 106) introduciendo el concepto de estructuras disipativas.

Estos dos acercamientos a las estructuras no lineales como son la autoorganización y las estructuras disipativas, fueron básicos para definir el patrón de vida que es la autopoiesis. Su significado etimológico es de: auto creación, en virtud que los seres vivos se caracterizan por ser independientes en cuanto a la autoproducción de sí mismos. Para que un sistema esté vivo su patrón de organización debe corresponder al de una red autopoiésica como lo indica (Capra, 1998, p. 219). Cabe hacer la aclaración que no todas las estructuras disipativas son sistemas vivos; por lo que no todas son redes autopoiésicas.

Gail Fleischaker biólogo y filosofo, se basó en tres criterios para resumir las propiedades de una red autopoiésica: Ser autolimitado, es decir que, la extensión del sistema queda delimitada por un perímetro que integra la red; ser auto generador significa que todos los componentes, incluyendo los del perímetro, son fruto de procesos de la red y ser auto perpetuante de modo que todos los componentes son continuamente reemplazados por los procesos de transformación del sistema según (Capra, 1998, p. 219).

Un punto interesante es que mientras Maturana y Varela hacen énfasis en la clausura organizativa del patrón de vida, por su parte llya Prigogine enfatiza la apertura de esta estructura al flujo de materia y energía, complementándose sus hallazgos. Concluyéndose entonces, que un sistema vivo posee dos condiciones: abierto estructuralmente, pero cerrado organizativamente. La materia y la energía fluyen a través de él, pero el sistema mantiene una forma estable y lo hace a través de su autoorganización y de manera autónoma como lo hace notar (Capra, 1998, p. 182).

Un elemento clave tanto de la autoorganización, la autopoiesis y las estructuras disipativas es la condición de estar alejados del equilibrio y aun así mantener una estructura relativamente estable en el tiempo, derivado de la recepción de energía externa para mantenerse, siendo su comportamiento no lineal. 
En la opinión de Maturana y Varela (1998) consideran que los sistemas autopoiésicos no funcionan todos de la misma manera, en virtud que pueden pertenecer a órdenes diferentes como se detalla a continuación:

1. Primer orden o moleculares en los que se encuentran las células.

2. Segundo orden que son agregados celulares y constituyen los organismos.

3. Tercer orden, que son los agregados de organismos como por ejemplo las colmenas, familias e incluso los sistemas sociales. (p. 18)

Cabe destacar que a los sistemas de tercer orden no los define la autopoiesis de sus componentes sino la relación de los organismos que los componen. En este orden de ideas, es necesario reconocer las diferencias propias de los sistemas de primer y segundo orden con los de orden superior o tercer orden, en virtud que muchos de los estilos de liderazgo dictatoriales y opresivos, han querido sustentar sus bases en la metáfora social de los sistemas vivos de acuerdo con (Capra, 1998, p. 222), situación que puede ser en esencia peligrosa para la salud de las sociedades y las personas.

En primera instancia en los organismos de primer y segundo orden se restringe la creatividad individual de sus miembros en virtud de garantizar su estabilidad estructural según (Capra, 1998, p. 224), ya que las partes existen para el todo y no al revés. Cuando se habla de sistemas sociales humanos, es diferente en virtud que los mismos existen para sus miembros, siendo necesario amplificar la creatividad individual. Cuando los Estados han adoptado posturas ideológicas opresivas, la calidad de vida de las personas ha sido reducida, así como su productividad menguada, resultando en períodos oscuros y tristes para la historia de la humanidad. La libertad para el ser humano es sinónimo de realización y plenitud de vida, sin embargo, la misma debe ser ejercida con responsabilidad y como indica Arriaga (2020) sin que la misma releve al ser humano de su responsabilidad de ejercer la libertad construyendo espacios para que ella pueda ser posible para todos sin excepción.

Otro aspecto a considerar es que las llamadas leyes de la naturaleza son quienes rigen el terreno físico, por su parte lo social está gobernado por normativa creada por el hombre mismo y a diferencia de lo natural, puede en un momento dado romperse como afirma (Capra, 1998, p. 222). Las sociedades pueden ir modificando sus normativas en función de necesidades (de hecho, lo hacen), para adaptarse a voluntad de sus miembros, cosa que no sucede en la naturaleza, ya que los organismos evolucionan para adaptarse según las leyes naturales, por ejemplo, la ley de la entropía, las cuales son inquebrantables. 
La trama de la vida como la denomina Fritjof Capra (1998) es un tejido, en donde confluyen lo vivo y lo no vivo. Siendo lo primero, creativo e impredecible, y por ende no lineal; por su parte, la condición sin vida en muchos de los casos puede ser lineal y estática (cabe aclarar que no en todos los casos como ejemplo están las estructuras disipativas). De lo que se desprende que las inter-retro-acciones en los ecosistemas al agregar el componente de vida pasan a ser complejas y por ende alejadas del equilibrio y deben estudiarse considerando esas condiciones.

Los hechos complejos no pueden explicarse desde la linealidad, en virtud que la no linealidad, le otorga matices, creatividad y sobre todo incertidumbre. Con ella tenemos que convivir diariamente, considerando las posibilidades infinitas, mucho más ricas que la realidad que es una sola.

En este orden de ideas, surge entonces el estudio de fenómenos económicos por ejemplo los cuales son complejos y deben considerar aspectos biológicos, físicos, sociales, culturales e incluso espirituales que inciden en ellos, situación que no se evidencia en muchos de los modelos económicos clásicos que son eminentemente matemáticos y por ende no responden a la realidad compleja y rica.

Otro abordaje de los procesos de autoorganización de la tierra lo presenta Lovelock en su Teoría Gaia en la cual describe al planeta como un organismo vivo que puede comprenderse mejor a través del estudio de sus procesos homeostáticos. Para tener una comprensión real de los fenómenos no podemos extraerlos de su contexto. El mundo entero constituye un ecosistema en el cual todos sus componentes tanto bióticos como abióticos, se relacionan e influyen unos en otros. El equilibrio solo existe en lo no vivo, donde no hay movimiento. Los fenómenos complejos son no lineales, y por ello merecen un tratamiento como tal son poco predecibles y capaces de evolucionar y crear. Lo que nos lleva a la necesidad de conocer las leyes que los rigen y sus patrones, en virtud de actuar consecuentemente para no romper la armonía dinámica de la vida como lo afirman (Castillo y Valdez, 2020; Girón, et al., 2019). Algo que es necesario enfatizar es que el patrón es el que da origen a las diversas relaciones entre las partes que conforman una estructura estable dentro de un proceso, por lo que sin el mismo los componentes carecen de vida.

\section{Conclusiones}

Los ecosistemas son estructuras alejadas del equilibrio, que disipan energía constantemente. Considerando la ley de la entropía, la energía que se disipa no se 
reconvierte, en forma que pueda ser reutilizada, por lo que el planeta como tal se está extinguiendo, es decir que conforme transcurre el tiempo se está consumiendo la misma hasta que ya no quede más de una forma útil. En esencia es una lucha contra el tiempo, más que una búsqueda de energía renovable per se. La reducción del costo energético de la humanidad debe ser una meta, así como optimizar el uso de la misma.

Basado en lo anterior, surge la necesidad de reflexionar en este punto sobre la responsabilidad de la ideología del capitalismo de crecimiento para la salud del planeta en general, en virtud, que no es una concepción que alargue la vida, sino que la reduce con el transcurso del tiempo.

Como seres humanos cuidamos nuestro cuerpo, lo alimentamos, nos ejercitamos, y buscamos mecanismos para reducir el estrés oxidativo, a pesar que indefectiblemente vamos a morir, sin embargo, intentamos extender nuestra vida través de acciones inteligentes, que hemos aprendido con la experiencia tanto propia como la acumulada por la especie humana. De la misma manera que lo hacemos con nuestro propio cuerpo, debemos hacerlo con la biosfera, no con una visión fatalista sino desde la perspectiva de la esperanza, la creatividad, espontaneidad y posibilidades infinitas que tenemos; en función de generar los cambios creativos para un planeta que funciona como un todo autoorganizado.

Como especie dependemos de los procesos homeostáticos del planeta y las repercusiones de nuestras acciones, sin importar el lugar donde vivamos afectan a todos, por lo que es necesario el desarrollo de una Conciencia Planetaria como le denomina Edgar Morín, en donde podamos cada uno hacernos responsables de las acciones pequeñas que suman y que cuentan con posibilidades infinitas de generar cambios que construyan entes plenos, saludables y armoniosos con el entorno.

\section{Declaración}

El estudio se realizó de acuerdo al Código de Ética y Buenas Prácticas COPE.

\section{Conflicto de intereses}

La autora declara no tener ningún conflicto de intereses. 


\section{Referencias}

Arriaga Ornelas, J. (2020). Convivencia: la via para generar dominios de libertad y las amenazas a su autopoiesis en la era digital. Contribuciones a las Ciencias Sociales. Obtenido de https://www.eumed.net/rev/cccss/2020/03/convivencia-era-digital.html.

Capra, F. (1998). La trama de la vida. Una nueva perspectiva de los sistemas vivos. Barcelona: Colección Anagrama.

Castillo López de Linares , N. C., \& Valdés Avila , I. M. . (2020). El biodesarrollo y su relación con los campos morfogenéticos. Revista Vida, Una Mirada Compleja, 2(1), 1-16. https://doi.org/10.36314/revistavida.v2i1.5

Girón Campos, B. D. T. ., Solis Fong, E. R. ., Retana Albanés, R. A. ., \& Gómez Marín, C. A. . (2019). VIDA: autopoiesis y complejidad: Plenitud de vida tras el lente de un mirar complejo. Revista Vida, Una Mirada Compleja, 1(1), 43-63. https://doi. org/10.36314/revistavida.v1i1.2

Maturana Romesin, H., \& Varela García, F. (1998). De máquinas y seres vivos. Autopoiesis: la organización de lo vivo. Chile: Editorial Universitaria.

Prigogine, I. (1997). El fin de las certidumbres. Barcelona: Andrés Bello.

\section{Sobre la autora \\ Nancy Carolina Castillo López}

Licenciada en Administración de Empresas, con una maestría en Docencia Superior Universitaria y actualmente estudia el Doctorado en Investigación en Educación en la Universidad de San Carlos de Guatemala. Ha trabajado en docencia desde el año 1998. Fungió durante algunos años como Asesora de Planificación Educativa de la Dirección Departamental de Educación de Chiquimula. Actualmente es catedrática en la Universidad de San Carlos de Guatemala, en carreras de licenciatura y de maestría. 
Copyright (c) Nancy Carolina Castillo López

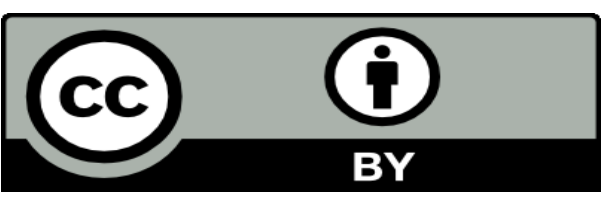

Este texto está protegido por una licencia CreativeCommons 4.0.

Usted es libre para compartir, copiar y redistribuir el material en cualquier medio o formato y adaptar el documento, remezclar, transformar y crear a partir del material para cualquier propósito, incluso comercialmente, siempre que cumpla la condición de atribución: usted debe reconocer el crédito de una obra de manera adecuada, proporcionar un enlace a la licencia, e indicar si se han realizado cambios. Puede hacerlo en cualquier forma razonable, pero no de forma tal que sugiera que tiene el apoyo del licenciante o lo recibe por el uso que hace. 